\title{
Pharmacokinetic Parameter Driven Outcomes Model Predicts a Reduction in Bleeding Events Associated with BAY 81-8973 Versus Antihemophilic Factor (Recombinant) Plasma/Albumin- Free Method in a Chinese Healthcare Setting
}

\section{Rong Chen}

Chengdu Third People's Hospital

Dmitry Gultyaev

Certara USA, Inc.

Johanna Lister

Formerly Certara USA, Inc

Rong Han

Bayer Healthcare Company. Ltd

$\mathrm{Nan} \mathrm{Hu}$

Bayer Healthcare Company. Ltd

Jean Malacan ( $\nabla$ jean.malacan@bayer.com )

Bayer Consumer Care AG

\section{Alexander Solms}

Bayer (Germany)

\section{Parth Vashi}

Formerly at US Data Generation and Observational Studies Bayer Corporation Jamie O’Hara

HCD Economics

Shanlian $\mathrm{Hu}$

Fudan University

\section{Research Article}

Keywords: Hemophilia A, Factor VIII products, pharmacokinetic, China, economic model, BAY 81-8973, rAHF-PFM 
DOI: https://doi.org/10.21203/rs.3.rs-1159955/v1

License: (c) (1) This work is licensed under a Creative Commons Attribution 4.0 International License. Read Full License 


\section{Abstract}

Background: Long-term prophylactic therapy is considered the standard of care for hemophilia A patients. This study models the long-term clinical and cost outcomes of two factor VIII (FVIII) products using a pharmacokinetic (PK) simulation model in a Chinese population.

Methods: Head-to-head PK profile data of BAY 81-8973 (KOVALTRY®) and antihemophilic factor (recombinant) plasma/albumin-free method (rAHF-PFM, ADVATE ${ }^{\circledR}$ ) were applied to a two-state (alive and dead) Markov model to simulate blood FVIII concentrations at a steady state in prophylactically-treated patients with hemophilia A. Worsening of the Pettersson score was simulated and decline was associated with the probability of having orthopaedic surgery. The only difference between the compounds was FVIII concentration at a given time; each subject was treated with $25 \mathrm{IU} / \mathrm{kg}$ every 3 days. The model used a lifetime horizon, with cycle lengths of 1 year.

Results: Cumulative bleeding events, joint bleeding events, and major bleeding events were reduced by $19.3 \%, 9.3 \%$ and $19.3 \%$, respectively for BAY $81-8973$ compared to rAHF-PFM. Hospitalizations and hospitalization days were also reduced by $19.3 \%$ for BAY $81-8973$ compared to rAHF-PFM. BAY 81-8973 resulted in both cost savings and a gain in quality adjusted life years (QALYs) compared to rAHF-PFM.

Conclusion: Based on modeled head-to-head comparisons, differences in PK-properties between BAY 818973 and rAHF-PFM result in a reduced number of bleeding events, leading to reduced costs and increased quality of life for BAY 81-8973. These results should be used to inform clinical practice in China when caring for patients with severe hemophilia A.

\section{Introduction}

Hemophilia is a group of rare hemorrhagic disorders, including mutations in the factor VIII (FVIII) gene (hemophilia A). A recent systematic review and meta-analysis undertaken across China estimated that the prevalence of hemophilia $A$ among males was 4.2 per 100,000 [1]. Patients with severe hemophilia $A$ experience repeated spontaneous bleeding caused by the deficiency of coagulation FVIII. Frequent bleeding in the joints can lead to joint damage, deformity, disability or negatively affect patients' quality of life [2]. Hemophilic arthropathy is the most common and severe complication observed in patients with hemophilia [3].

Prophylaxis is considered the standard of care for hemophilia patients, as it reduces the morbidity and increases the quality of life of patients [4]. Compared to on-demand treatment, prophylactic treatment can reduce joint bleeding and the development of hemophilic arthropathy [5]. Without prophylactic FVIII product replacement therapy, patients may experience repeated bleeds into the same joints, resulting in significant health-related quality of life deterioration [6].

The World Federation of Hemophilia (WFH) states that prophylaxis is always recommended over episodic therapy. While Chinese guidelines explicitly state that diagnosed patients with hemophilia A should 
receive treatment, with FVIII products as the recommended treatment of choice, there is currently no consensus on long-term prophylactic therapy in adult patients [7]. In countries with adequate resources, standard-dose FVIII product prophylaxis is the standard care for hemophilia patients [4]. However, in developing countries such as China, low-dose prophylactic therapy and on-demand therapy remain the primary method of treatment [3], though the mean per capita factor VIII product use in 2018 was 0.026 $\mathrm{IU} /$ total population, which is lower than other countries in the region with similar economic profiles [8]. While Chinese guidelines do not state a preferred prophylactic therapy, they recognize that short-term prophylaxis with high dose can reduce bleeding and improve quality of life [7].

BAY 81-8973 (KOVALTRY®) is a full-length recombinant FVIII product. A phase I, open-label, crossover study [9] compared the pharmacokinetic (PK) profile of BAY 81-8973 with antihemophilic factor (recombinant) plasma/albumin-free method (rAHF-PFM, ADVATE $®$ ) among men aged 18-65 years with severe hemophilia $A$ and $\geq 150$ exposure days to FVIII product and found that BAY 81-8973 exhibited a superior pharmacokinetic (PK) profile [9]. The PK of coagulation factors varies across populations;[10] therefore, a model integrating the long-term clinical and cost outcomes of BAY 81-8973 prophylaxis versus rAHF-PFM was developed in a Chinese population. The impacts on HRQoL, along with incremental costs, and the associated incremental cost-effectiveness ratio (ICER) are reported in order to facilitate health policy decision-making for this population.

\section{Materials And Methods}

\section{Model Overview}

A two-state (alive and dead) Markov model was developed in Microsoft Excel to assess the costeffectiveness of FVIII prophylaxis in adult men ( $\geq 18$ years) with severe hemophilia A from the perspective of the Chinese health care system. The model simulates the blood FVIII concentrations at a steady state following prophylaxis treatment with either BAY 81-8973 or rAHF-PFM in a cohort of 10,000 patients. All patients entered the Markov model within the "alive" state (represented by the top half of the model structure) with the number of bleeding events determined by the model; note that within this model, bleeds are transitory and are not considered a health state (Figure 1).

Within the alive state, patients were treated prophylactically with either BAY 81-8973 or rAHF-PFM, with the only difference between the two FVIII products being the compounds PK profile, resulting FVIII concentrations at a given time. The probability of bleeds, along with the number of bleeds, is then determined. Bleeds determine the Pettersson score, which results in the estimation of quality of life. Patients can transition to death at any time through general population background mortality. Further details on the model structure can be found in the Additional File 1. A lifetime time horizon, with cycle lengths of one year, was used. A discount rate of $5.0 \%$ was applied to costs and effects in the base case. [12] Patients were followed until death.

\section{Model inputs}


Arthropathy development and joint replacement surgery in the model were driven by increases in the Pettersson Score, a validated radiological scoring system which ranges from 0 for joints without signs of arthropathy to a high of 78 points [17]. Patients in each bleeding-per-year category accumulated joint bleeds over time, and based on the cumulative value, the Pettersson score was determined, with an estimation of the associated joint deterioration. Patients at baseline were assumed to have had 85 joint bleeds [18] and an increase of 12.6 joint bleeds was assumed to correlate to a one point increase in the Pettersson score (95\% Cl: 11.1 - 14.7) [19]. A Pettersson score of 28 was assumed to represent clinicallyrelevant damage that required orthopaedic (joint replacement) surgery [18]. Worsening of the Pettersson score was simulated and clinically relevant decline was associated with the probability of having orthopedic surgery. Change in Pettersson score is a valid approach to projecting long-term outcomes [20].

The population-specific data on mortality in patients with severe hemophilia A is not available in China. In the absence of this information, a standardized mortality ratio was applied to the probability of death reflected general male age-adjusted mortality in China, based on the global data that hemophilia $\mathrm{A}$ patients have a life expectancy of about 10 years or less [21]. A sensitivity analysis assuming equivalent life expectancy between treated hemophilia A patients and that of the general population was also explored [22]. To account for the increased risk in death following major surgery or bleed, the probability of death in the year following major orthopaedic surgery or major bleed was increased by an additional $1 \%$ [23].

Utility values were health-state specific and are presented in Table 1. 
Table 1

Utility and cost values, base case analysis

\section{Utilities}

State Mean (SD) Source

Pettersson score 0-4 $0.82( \pm 0.13) \quad$ Fischer, K. et al.[6]

Pettersson score 5-12 $\quad 0.81( \pm 0.12)$

Pettersson score 13-21 $0.77( \pm 0.13)$

Pettersson score 22-39 $0.74( \pm 0.12)$

Pettersson score 40-78 $0.72( \pm 0.11)$

Orthopaedic surgery $\quad-0.39( \pm 0.04)$

Ballal, R. D. et al.[31] Utility decrement is assumed to last for 30.4 days. Standard deviation not reported, 10\% assumed

Hospitalisation due to $\quad-0.39( \pm 0.04)$

Assumption. Utility decrement is assumed to be the major bleed same as for orthopaedic surgery but for a shorter time period (3.6 days).

\section{Costs}

Input Mean (CNY) Source

BAY 81-8973 per IU

4.488

Huo et al.[32]

rAHF-PFMper IU

4.488

Huo et al.[32]

Hospitalization for major bleed

74,122

Expert opinion, based on survey

Physician visit 500

Expert opinion, based on survey

Cost of orthopaedic surgery

300,465

Expert opinion, based on survey

Rehabilitation costs

937.50

Expert opinion, based on survey

Total compensation per $\quad 25.40$

hour

\section{Resource Use}

Input

Unit/Frequency Source

Extra dose for treatment of bleed $24.11 \mathrm{IU} / \mathrm{kg} \quad$ Calculated BAY 81-8973

${ }^{\dagger}$ Disutility of bleed into target joint was -0.120 (SD: 0.026). 


\section{Utilities}

Extra dose for
treatment of bleed -
rAHF-PFM

Doctor visits (with or $\quad 12$ / year $\quad$ Expert opinion
without joint bleeds)

Retirement age $\quad 60 \quad$ China labour statistics[34]

Full time employment $\quad 45.6 \% \quad$ Sun et al.[35]

Missing days of work, $\quad 3.40 \quad$ Chen et al. 2017[11] annual

${ }^{\dagger}$ Disutility of bleed into target joint was -0.120 (SD: 0.026).

rAHF-PFM: antihemophilic factor (recombinant) plasma/albumin-free method

The utility associated with arthropathy was based on the Pettersson score [17]. Major bleeds associated with hospitalisation were assumed to have the same utility deterioration as for orthopaedic surgery. The duration of a major bleed was assumed to be 3.6 days which was validated by a Chinese clinical expert involved in the study herein.

Direct costs in the model included costs of the following: prophylaxis treatment, hospitalisation for treating major bleeds, cost of physician visit (with or without bleeds), and cost of orthopaedic surgery (Table 1). The dose assumed in the model was $25 \mathrm{IU} / \mathrm{kg}$ every 3 days.

In absence of published data, costs for hospitalization for major bleed, physician visits, orthopaedic surgery and rehabilitation were taken from a survey conducted in 2016 among five physicians in five different hemophilia treatment centers that were selected across four major cities in China (Beijing in North China, Guangzhou in South China, Chengdu in West China and Wuhan in Central China). Lost productivity due to missing work was included in the model. This was calculated based on the estimated proportion of males who were employed, the estimated annual number of days missed of work per year, and the average total compensation per hour. Unit costs are reported in 2020 Chinese yuan (CNY).

\section{Outcomes}

Based on differences in PK between BAY 81-8973 and rAHF-PFM, the model evaluated number of bleeding events, total costs and quality-adjusted life-years. The ICER was calculated as the total incremental costs divided by the total incremental quality-adjusted life years. The base case analysis is presented from the public payer perspective; scenario analyses from a societal perspective, accounting for indirect costs due to the productivity losses experienced by both patients and caregivers, is also presented. 
Both a probabilistic and deterministic sensitivity analyses were undertaken to assess parameter uncertainty. Multiple scenario analyses were considered including varying the dosing to $20 \mathrm{lU} / \mathrm{kg}$ and 15 $\mathrm{IU} / \mathrm{kg}$, given the lower doses use observed in China [24], varying the number of joint bleeds at baseline (lower value of 42.5 and upper value of 170), as well as a separate source of utility values identified in a hemophilia A review from the Institute of Clinical and Economic Review given the sparse amount of data available in China specifically and the relevance of US frameworks in other country contexts [25].

\section{Results}

\section{FVIII concentration}

The FVIII concentrations (with their 95\% prediction intervals) are presented in Figure 2.

As the elimination phase was longer for BAY 81-8973 with a longer half-life, FVIII concentrations were maintained above $1 \mathrm{IU} / \mathrm{mL}$ over a longer period of time. Time to achieve $1 \mathrm{IU} / \mathrm{mL}$ were 68.3 hours and 60.7 hours after dosing for BAY 81-8973 and rAHF-PFM, respectively.

\section{Bleeding events}

Cumulative bleeding events, cumulative joint bleeding events and cumulative major bleeding events, over 12 months, are presented in Figure 3 for BAY 81-8973 and rAHF-PFM.

Compared to rAHF-PFM, treatment with BAY 81-8973 resulted in an overall decrease of $19.2 \%, 10.2 \%$ and $20.0 \%$ in cumulative bleeding events, cumulative joint bleeding events and cumulative major bleeding events, respectively.

\section{Hospitalizations and orthopaedic surgeries}

There were a total of 0.3 hospitalizations with a mean of 0.3 hospitalization days for BAY 81-8973 compared to 0.4 hospitalizations with a mean of 0.4 hospitalization days for rAHF-PFM over a lifetime. This represents a reduction in hospitalizations of $19.3 \%$ for treatment with BAY 81-8973 compared to rAHF-PFM. Orthopaedic surgeries were equally lower for BAY 81-8973 versus rAHF-PFM: 0.05 vs 0.07, representing a decrease of $31.6 \%$.

\section{Pettersson score}

Average Pettersson score increased with increasing age, over the lifetime of the cohort, but was lower for those receiving BAY 81-8973 (10.9) compared to rAHF-PFM (12.3).

\section{Incremental cost-effectiveness results}

Costs and QALYs by health state are presented in Table 2 . 
Table 2

Cost-effectiveness results, base case analysis

\begin{tabular}{|llll|}
\hline & BAY 81-8973 & rAHF-PFM & Incremental \\
\hline Drug/Procedure cost & $¥ 16,329,777$ & $¥ 16,325,648$ & $¥ 4,129$ \\
\hline Bleeding related costs & $¥ 486,877$ & $¥ 612,852$ & $-¥ 125,974$ \\
\hline Physician visits & $¥ 108,348$ & $¥ 108,321$ & $¥ 27$ \\
\hline Societal cost & $¥ 5,388$ & $¥ 5,390$ & $-¥ 2$ \\
\hline Total cost & $¥ 16,930,390$ & $¥ 17,052,210$ & $¥ 121,819$ \\
\hline QALYs & 13.220 & 13.167 & 0.053 \\
\hline Life years & 18.058 & 18.053 & 0.005 \\
\hline ICER & BAY 81-8973 is dominant (lower costs and higher QALY gain) \\
\hline $\begin{array}{l}\text { ICER: incremental cost-effectiveness ratio; rAHF-PFM: antihemophilic factor (recombinant) } \\
\text { plasma/albumin-free method; QALYs: quality-adjusted life year }\end{array}$ \\
\hline
\end{tabular}

Prophylactic treatment with BAY 81-8973 resulted in a cost savings of $¥ 121,819$, mainly due to savings in bleeding related costs over the total lifetime of the patient. BAY 81-8973 compared to rAHF-PFM also resulted in 0.053 incremental QALYs. This resulted in BAY 81-8973 being a dominant strategy over rAHFPFM (less costly and more effective).

\section{Sensitivity analyses}

Figure 4 presents the one-way deterministic sensitivity analyses. The model was most sensitive to utility values for Pettersson scores $13-21$ and $22-39$, followed by discounting.

When all parameters were varied simultaneously, BAY 81-8973 was less costly and cost-effective in over $95 \%$ of the scenarios compared to rAHF-PFM.

Scenario analyses of using lower prophylaxis dosing every three days were explored. As dosing decreased from the base case of $25 \mathrm{IU} / \mathrm{kg}$ to $20 \mathrm{IU} / \mathrm{kg}$ and $15 \mathrm{IU} / \mathrm{kg}$, BAY 81-8973 remains cost-effective compared to rAHF-PFM as presented (Table 3); however, the absolute effectiveness of BAY 81-8973 is greatest with the highest dose. 
Table 3

Scenario analyses of lower dosing

\section{Dosing $25 \mathrm{IU} / \mathrm{kg}$ (base case) \\ Dosing $20 \mathrm{lU} / \mathrm{kg}$ \\ Dosing $15 \mathrm{IU} / \mathrm{kg}$}

Costs

\begin{tabular}{llll} 
BAY 81-8973 & $¥ 16,930,390$ & $¥ 13,703,193$ & $¥ 10,487,104$ \\
\hline rAHF-PFM & $¥ 17,052,210$ & $¥ 13,821,831$ & $¥ 10,599,846$ \\
\hline Incremental cost & $¥ 121,819$ & $¥ 118,638$ & $¥ 112,742$ \\
\hline QALYs & & & \\
\hline BAY 81-8973 & 13.220 & $13.202^{\dagger}$ & $13.179^{\dagger}$ \\
\hline rAHF-PFM & 13.167 & $13.151^{\dagger}$ & $13.132^{\dagger}$ \\
\hline Incremental QALYs & $\mathbf{0 . 0 5 3}$ & $\mathbf{0 . 0 5 0}$ & 0.047 \\
\hline ICER & BAY 81-8973 is dominant (lower costs and higher QALY gain)
\end{tabular}

${ }^{\dagger}$ Derived using the annual bleeding rate of $25 \mathrm{IU} / \mathrm{kg}$

ICER: incremental cost-effectiveness ratio; rAHF-PFM: antihemophilic factor (recombinant) plasma/albumin-free method; QALYs: quality-adjusted life years

Varying the number of joint bleeds at baseline or the utilities had negligible impact on the results compared to the base case analysis.

\section{Discussion}

Hemophilia A results in substantial morbidity and the World Federation of Hemophilia recommends management with prophylaxis as part of standard of care. Given individual variability in response to treatment of FVIII concentrates in patients with hemophilia A, PK-tailored prophylaxis is being proposed and considered as a more efficient approach for treatment with FVIII products. This study assessed the impact of prophylactic treatment with BAY 81-8973 and rAHF-PFM on health-related quality of life and costs for patients with severe hemophilia A in China, using a PK model. This model found that prophylactic treatment with BAY 81-8973 resulted in cost savings given the reduction in bleeding. Therefore BAY 81-8973 is a cost-effective option as compared to rAHF-PFM, across all doses; however, total QALY gain and incremental QALYs were higher with the highest dose of $25 \mathrm{IU} / \mathrm{kg}$ every 3 days.

These results are aligned with previous PK studies, where BAY 81-8973 was shown to have better PK characteristics, as measured by slower clearance, compared to rAHF-PFM [26]. A PK modelling study undertaken in China in pediatric patients found that annual bleeding rates and annual joint bleeding rates decreased in patients switching from rAHF-PFM to BAY 81-8973 [27]. Similar clinical outcomes were observed in adult patients, that found that the annual bleeding rate, and the annual joint bleeding rate was reduced with prophylactic treatment with BAY 81-8973 [28]. The incremental QALY gain in this study 
of 0.02 is also found to be greater than that identified in a cost-effectiveness models of other prophylactic treatments vs standard of care [25].

Relationship between actual FVIII concentration and bleedings were analyzed by means of a repeated time to event approach based on data obtained in the clinical development program of BAY 81-8973 (Leopold I [14]) given 20-50 IU/kg 2-3 times per week for the duration of the study. In this regard, simulations beyond the dose range investigated and the observed treatment duration have less validity. For instance, long-term treatment effects (e.g. improvement of joint status) beyond the effect of actual FVIII level on bleeding risk could not be described based on the underlying data. However, as this is assumed to equally impact treatment with BAY 81-8973 and rAHF-PFM, the overall conclusion won't be affected by such limitations.

This study has limitations. The presented results focus on a constant treatment over the lifetime of a patient of patients receiving prophylaxis. Episodes of non-compliance or changes to a given treatment schedule over time, which is likely more reflective of a real-world patient experience, were not considered. However, as such variations in the treatment schedule would affect both treatment arms, the model outcomes would be affected equally and the omission of this does not impact the results observed. To that end, this model does not explore the impact of prophylaxis vs no prophylaxis. In the absence of data from on-demand treatment or placebo, this study is not able to estimate the connection between FVIII and bleeding corresponding to untreated patients; these results cannot be applied to treatment strategies other than prophylaxis. Further, no covariates were included in the model to adjust for differences in patient baseline characteristics. The yearly bleeding rate was also assumed to remain constant over time. In order to explore the scenarios of lower doses, the bleeding rate in the population receiving $25 \mathrm{IU} / \mathrm{kg}$ was used as a proxy for $20 \mathrm{IU} / \mathrm{kg}$ and $15 \mathrm{IU} / \mathrm{kg}$ as dose ranges were not extensively studied in the LEOPOLD trial [14]; scenario analyses of lower doses should be interpreted with caution. Finally, every effort was made to source inputs in a Chinese population, however, this was not always available. Inputs that may not be generalizable to a Chinese population include the utilities used in this model, the proportion of bleeds that occurred in the joints and the proportion of bleeds that were considered major.

\section{Conclusion}

This is the first cost-effectiveness model of adult patients with hemophilia A in China treated prophylactically with FVIII product. Given the high cost of caring for severe hemophilia A patients with a chronic, prophylaxis treatment, optimizing the costs and benefits of various treatment approaches is of paramount importance to payers in China. This PK model demonstrated that BAY 81-8973 is a cost saving and cost effective option, due to reductions in bleeding events along with an increase quality of life, highlighting the value of BAY 81-8973 for prophylactic treatment of patients with severe hemophilia A in China.

\section{Declarations}




\section{Declaration of Data Use:}

Data was utilized from population census of the People's Republic of China. National Bureau of Statistics of China: China Statistics Press, 2012. These data is from the latest life tables and the reference 21 from our paper can be accessed via follow link:

http://www.stats.gov.cn/tjsj/pcsj/rkpc/6rp/indexch.htm

This is the official website of China National Bureau of Statistics. When you open the link, please scroll

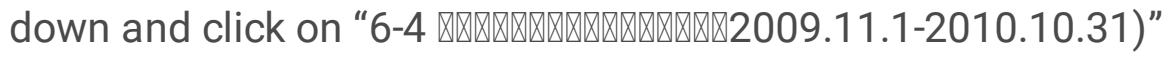

All data generated or analysed during this study are included in this published article and therefore, all methods were performed in accordance with the relevant guidelines and regulations. There is no additional data to be disclosed or shared.

- Ethics approval and consent to participate: N/A

- Consent for publication: N/A

- Availability of data and materials: N/A

- Competing interests: N/A

- Funding: Financial support for this manuscript was provided by Bayer

- Authors' contributions: JM designed the research study. DG, JL, RH, NH, AS, PV, JO'H performed the research. DG, AS, PV, JOH analysed the data. All authors contributed to the development of the manuscript, reviewed and commented on each draft, and approved the final draft.

- Acknowledgements: N/A

- Authors' information (optional)

\section{References}

1. Qu Y, Nie X, Yang Z, Yin H, Pang Y, Dong P, et al. The prevalence of hemophilia in mainland China: a systematic review and meta-analysis. The Southeast Asian journal of tropical medicine and public health. 2014;45(2):455-66.

2. [Consensus of Chinese expert on the diagnosis and treatment of hemophilia (version 2017)]. Zhonghua xue ye xue za zhi = Zhonghua xueyexue zazhi. 2017;38(5):364-70.

3. Liu S, Zhou RF, Jin ZB, Wu M, Zhang PY. Age-related severity and distribution of haemophilic arthropathy of the knee, ankle and elbow among Chinese patients with haemophilia. Haemophilia: the official journal of the World Federation of Hemophilia. 2020;26(1):129-35.

4. Srivastava A, Santagostino E, Dougall A, Kitchen S, Sutherland M, Pipe SW, et al. WFH Guidelines for the Management of Hemophilia, 3rd edition. Haemophilia: the official journal of the World Federation of Hemophilia. 2020;26 Suppl 6:1-158. 
5. Iorio A, Marchesini E, Marcucci M, Stobart K, Chan AK. Clotting factor concentrates given to prevent bleeding and bleeding-related complications in people with hemophilia A or B. The Cochrane database of systematic reviews. 2011(9):Cd003429.

6. Fischer K, de Kleijn P, Negrier C, Mauser-Bunschoten EP, van der Valk PR, van Galen KP, et al. The association of haemophilic arthropathy with Health-Related Quality of Life: a post hoc analysis. Haemophilia: the official journal of the World Federation of Hemophilia. 2016;22(6):833-40.

7. [Chinese guidelines on the treatment of hemophilia (version 2020)]. Zhonghua xue ye xue za zhi = Zhonghua xueyexue zazhi. 2020;41(4):265-71.

8. World Federation of Hemophilia. Report on the Annual Global Survey: 20th Anniversary. 2018.

9. Shah A, Solms A, Garmann D, Katterle Y, Avramova V, Simeonov S, et al. Improved Pharmacokinetics with BAY 81-8973 Versus Antihemophilic Factor (Recombinant) Plasma/Albumin-Free Method: A Randomized Pharmacokinetic Study in Patients with Severe Hemophilia A. Clinical pharmacokinetics. 2017;56(9):1045-55.

10. Iorio A. Using pharmacokinetics to individualize hemophilia therapy. Hematology Am Soc Hematol Educ Program. 2017;2017(1):595-604.

11. Chen CX, Baker JR, Nichol MB. Economic Burden of Illness among Persons with Hemophilia B from HUGS Vb: Examining the Association of Severity and Treatment Regimens with Costs and Annual Bleed Rates. Value in health: the journal of the International Society for Pharmacoeconomics and Outcomes Research. 2017;20(8):1074-82.

12. China Guidelines for Pharmacoeconomics Evaluations. Gordon G Liu, editor: China Market Press; 2020.

13. Abrantes J, Solms A, Garmann D, Nielsen E, Jonsson S, Karlsson M, editors. Integrated modelling of factor VIII activity kinetics, occurrence of bleeds and individual characteristics in haemophilia A patients using a full random effects modelling (FREM) approach. PAGE meeting; 2018; Montreux, Switzerland.

14. Saxena K, Lalezari S, Oldenburg J, Tseneklidou-Stoeter D, Beckmann H, Yoon M, et al. Efficacy and safety of BAY 81-8973, a full-length recombinant factor VIII: results from the LEOPOLD I trial. Haemophilia: the official journal of the World Federation of Hemophilia. 2016;22(5):706-12.

15. Tarantino MD, Collins PW, Hay CR, Shapiro AD, Gruppo RA, Berntorp E, et al. Clinical evaluation of an advanced category antihaemophilic factor prepared using a plasma/albumin-free method: pharmacokinetics, efficacy, and safety in previously treated patients with haemophilia A. Haemophilia: the official journal of the World Federation of Hemophilia. 2004;10(5):428-37.

16. Witmer C, Presley R, Kulkarni R, Soucie JM, Manno CS, Raffini L. Associations between intracranial haemorrhage and prescribed prophylaxis in a large cohort of haemophilia patients in the United States. British journal of haematology. 2011;152(2):211-6.

17. Pettersson H, Nilsson IM, Hedner U, Noréhn K, Ahlberg A. Radiologic evaluation of prophylaxis in severe haemophilia. Acta paediatrica Scandinavica. 1981;70(4):565-70. 
18. Fischer K, Pouw ME, Lewandowski D, Janssen MP, van den Berg HM, van Hout BA. A modeling approach to evaluate long-term outcome of prophylactic and on demand treatment strategies for severe hemophilia A. Haematologica. 2011;96(5):738-43.

19. Fischer K, van Hout BA, van der Bom JG, Grobbee DE, van den Berg HM. Association between joint bleeds and pettersson scores in severe haemophilia. Acta Radiologica. 2002;43(5):528-32.

20. Fischer K, Poonnoose P, Dunn AL, Babyn P, Manco-Johnson MJ, David JA, et al. Choosing outcome assessment tools in haemophilia care and research: a multidisciplinary perspective. Haemophilia: the official journal of the World Federation of Hemophilia. 2017;23(1):11-24.

21. National Bureau of Statistics of China. 2010 population census of the People's Republic of China. 2012.

22. Reitter S, Waldhoer T, Vutuc C, Lechner K, Pabinger I. Survival in a cohort of patients with haemophilia at the haemophilia care center in Vienna, Austria, from 1983 to 2006. Haemophilia: the official journal of the World Federation of Hemophilia. 2009;15(4):888-93.

23. Miners AH, Sabin CA, Tolley $\mathrm{KH}$, Lee CA. Cost-utility analysis of primary prophylaxis versus treatment on-demand for individuals with severe haemophilia. PharmacoEconomics. 2002;20(11):759-74.

24. Song X, Liu W, Xue F, Zhong J, Yang Y, Liu Y, et al. Real-world analysis of haemophilia patients in China: A single centre's experience. Haemophilia: the official journal of the World Federation of Hemophilia. 2020;26(4):584-90.

25. Rind D, Walton S, Agboola F, Herron-Smith S, Quach D, Chapman R, et al. Valoctocogene Roxaparvovec and Emicizumab for Hemophilia A: Effectiveness and Value; Final Report.; 2020.

26. Shah A, Delesen H, Garger S, Lalezari S. Pharmacokinetic properties of BAY 81-8973, a full-length recombinant factor VIII. Haemophilia: the official journal of the World Federation of Hemophilia. 2015;21(6):766-71.

27. Huang K, Li G, Zhen Y, Wu X, Chen Z, Wu R. Comparative pharmacokinetics of Kogenate FS and Kovaltry in 14 Chinese paediatric patients with haemophilia A: A single-centre study. Haemophilia: the official journal of the World Federation of Hemophilia. 2020.

28. Megías-Vericat JE, Bonanad S, Haya S, Cid AR, Marqués MR, Monte-Boquet E, et al. Cross-sectional comparative study of pharmacokinetics and efficacy between sucrose-formulated recombinant factor VIII (Kogenate $\left({ }^{\circledR}\right)$ ) and BAY 81-8973 (Kovaltry $\left({ }^{\circledR}\right)$ ) in patients with severe or moderate haemophilia A in prophylaxis. Haemophilia: the official journal of the World Federation of Hemophilia. 2019;25(3):e215-e8.

29. Neufeld EJ, Recht M, Sabio H, Saxena K, Solem CT, Pickard AS, et al. Effect of acute bleeding on daily quality of life assessments in patients with congenital hemophilia with inhibitors and their families: observations from the dosing observational study in hemophilia. Value in health: the journal of the International Society for Pharmacoeconomics and Outcomes Research. 2012;15(6):916-25.

30. Mazza G, O'Hare J, Carroll L, Camp C, Hoxer C, Wilkinson L. The impact of haemophilia complications on health-related quality of life for adults with severe haemophilia.: Value in Health; 2016. 
31. Ballal RD, Botteman MF, Foley I, Stephens JM, Wilke CT, Joshi AV. Economic evaluation of major knee surgery with recombinant activated factor VII in hemophilia patients with high titer inhibitors and advanced knee arthropathy: exploratory results via literature-based modeling. Current medical research and opinion. 2008;24(3):753-68.

32. Huo J, Zhao Z. [Evaluation of Safety and Pharmacological Characteristics in Vivo of Recombinant Human Coagulation Factor VIII]. Drug Evaluation. 2019;16:3-7.

33. National Bureau of Statistics of China. China Statistical Yearbook 2019 [Available from: http://www.stats.gov.cn/tjsj/ndsj/2019/indexch.htm.

34. National Bureau of Statistics of China. China Labour Statistical Yearbook 2016 [Available from: http://www.mohrss.gov.cn/2016/indexeh.htm.

35. Sun J, Zhao Y, Yang R, Guan T, lorio A. The demographics, treatment characteristics and quality of life of adult people with haemophilia in China - results from the HERO study. Haemophilia: the official journal of the World Federation of Hemophilia. 2017;23(1):89-97.

\section{Figures}

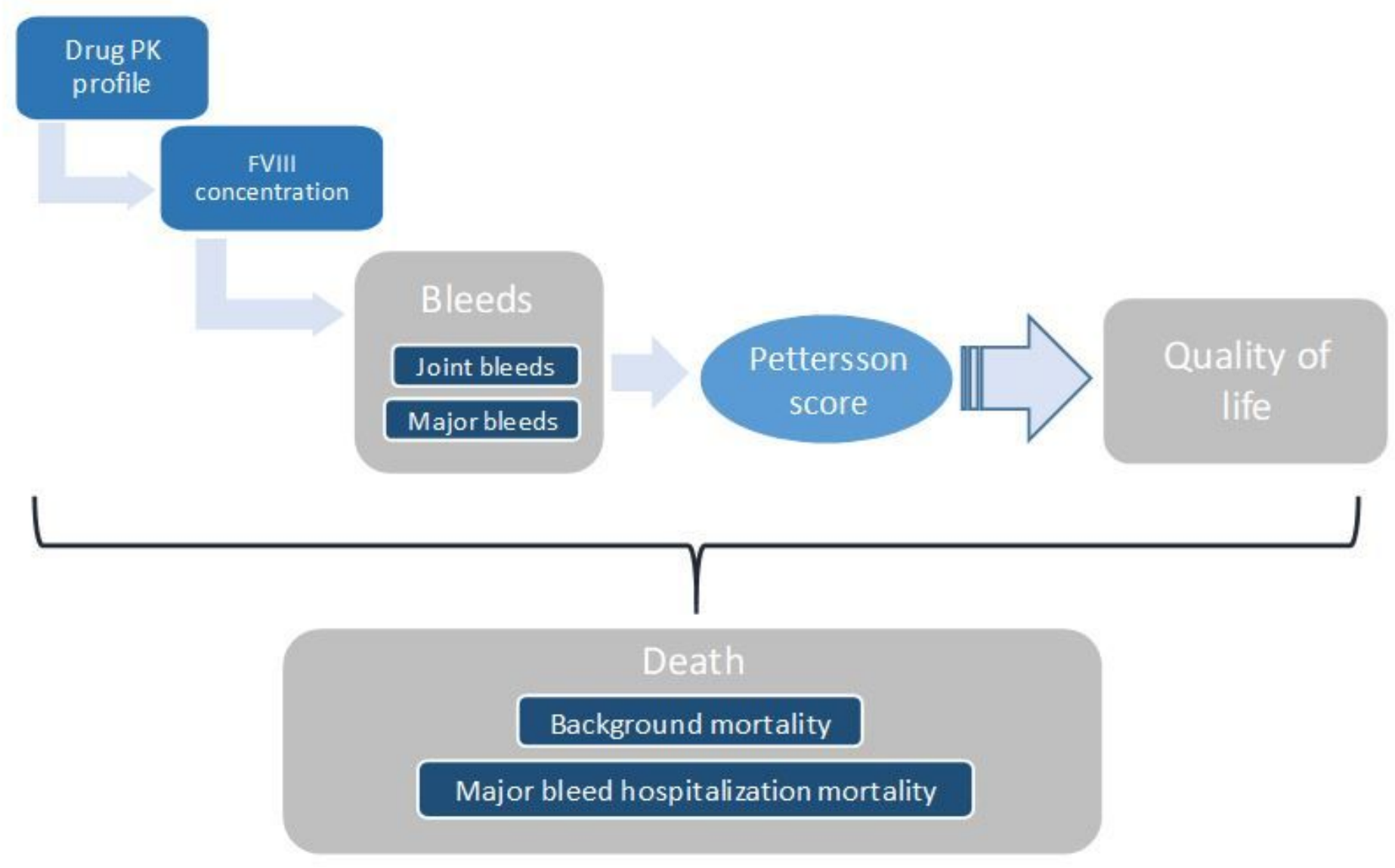

Figure 1

Overview of model structure 


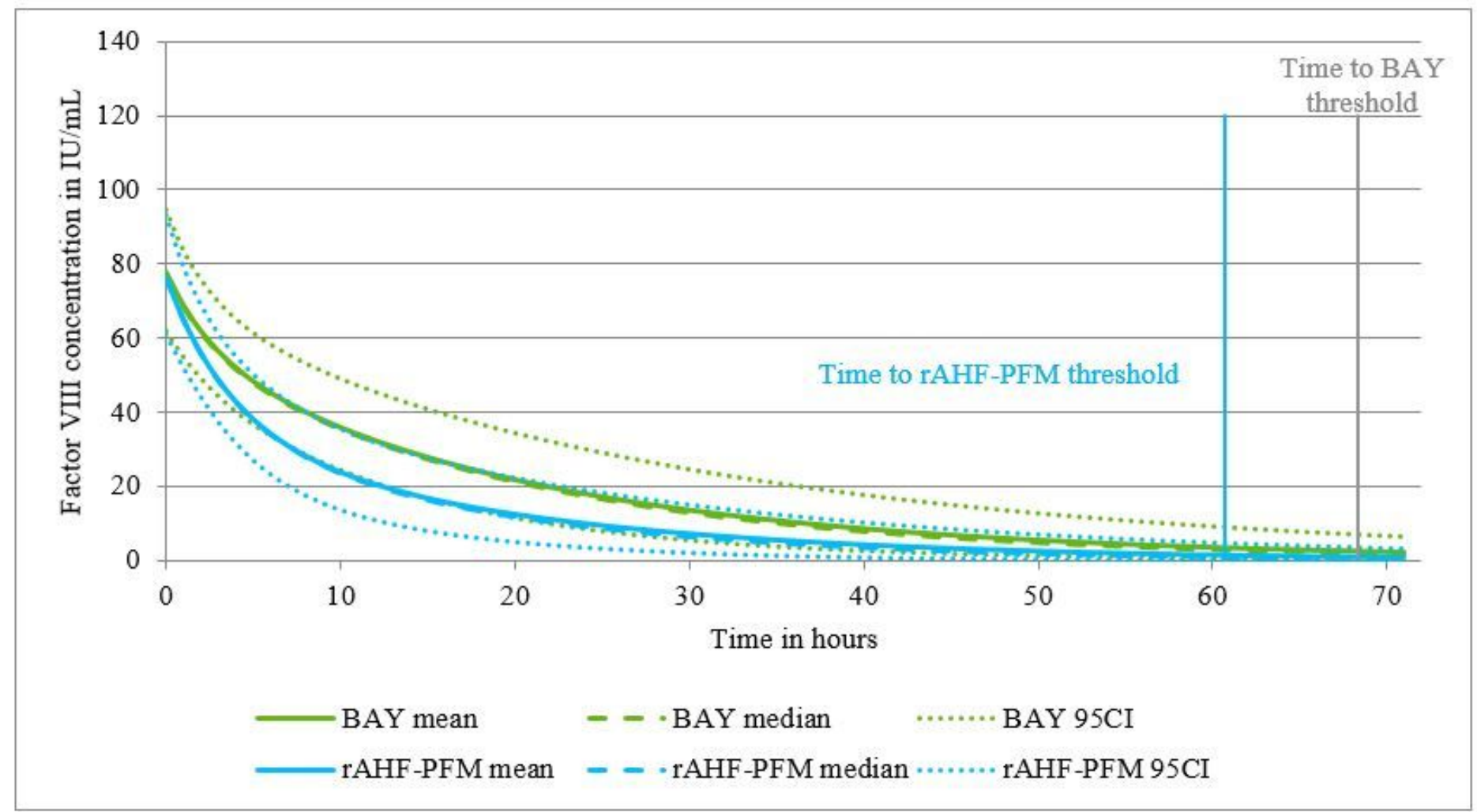

95CI: 95\% confidence interval; rAHF-PFM: antihemophilic factor (recombinant) plasma/albumin-free method; BAY: BAY $81-8973$

Figure 2

FVIII concentrations over time 


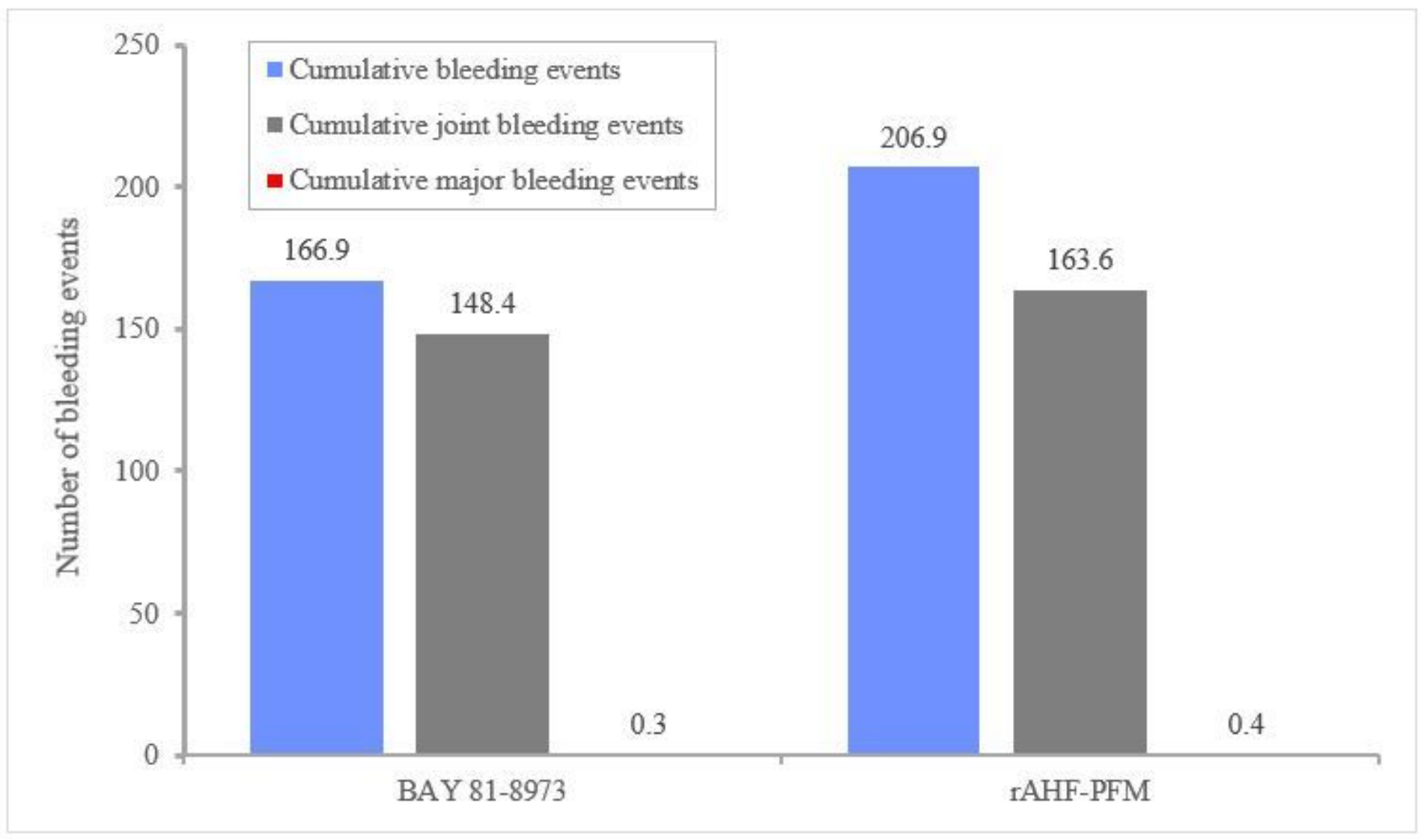

rAHF-PFM: antihemophilic factor (recombinant) plasma/albumin-free method; BAY: BAY 81-8973

\section{Figure 3}

\section{Bleeding events}

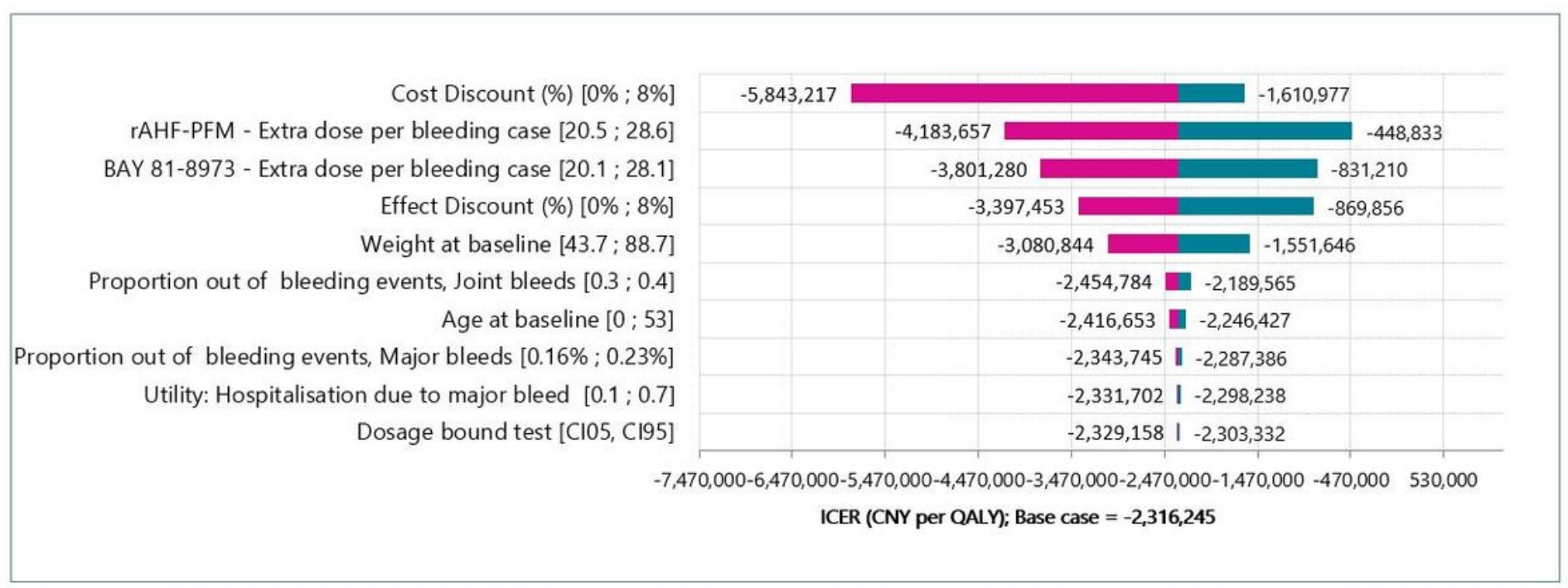

rAHF-PFM: antihemophilic factor (recombinant) plasma/albumin-free method

\section{Figure 4}




\section{Tornado diagram}

\section{Supplementary Files}

This is a list of supplementary files associated with this preprint. Click to download.

- AdditionalFile1.docx 